PROCEEDINGS OF THE

AMERICAN MATHEMATICAL SOCIETY

Volume 136, Number 12, December 2008, Pages 4385-4388

S 0002-9939(08)09610-X

Article electronically published on July 23, 2008

\title{
A SHORT PROOF OF HARA AND NAKAI'S THEOREM
}

\author{
BYUNG-GEUN OH
}

(Communicated by Mario Bonk)

\begin{abstract}
We give a short proof of the following theorem of Hara and Nakai: for a finitely bordered Riemann surface $R$, one can find an upper bound of the corona constant of $R$ that depends only on the genus and the number of boundary components of $R$.
\end{abstract}

\section{THE CORONA PROBLEM AND HARA-NAKAI's THEOREM}

For a given Riemann surface $R$, let $H^{\infty}(R)$ denote the uniform algebra of bounded analytic functions on $R$. To avoid pathological cases, we also assume that $H^{\infty}(R)$ separates the points in $R$; i.e., for any $x_{1}, x_{2} \in R, x_{1} \neq x_{2}$, there exists a function $f \in H^{\infty}(R)$ with $f\left(x_{1}\right) \neq f\left(x_{2}\right)$.

We next consider the maximal ideal space $\mathcal{M}(R)$ of $H^{\infty}(R)$, and observe that each $M \in \mathcal{M}(R)$ can be identified with $\varphi_{M}: H^{\infty}(R) \rightarrow \mathbb{C}$, where $\varphi_{M}$ is the complex homomorphism that has $M$ as its kernel. This means that the maximal ideal space $\mathcal{M}(R)$ can be regarded as a subspace of the dual space $\left(H^{\infty}(R)\right)^{*}$ of $H^{\infty}(R)$. Moreover, it also implies that we can equip $\mathcal{M}(R)$ with the Gelfand topology; thus $\mathcal{M}(R)$ can be thought of as a closed subspace of $\left(H^{\infty}(R)\right)^{*}$ that is contained in the unit sphere. For the details, see for example Chap. V-1 of [10].

We know that each $\xi \in R$ corresponds to the maximal ideal

$$
M_{\xi}=\left\{f \in H^{\infty}(R): f(\xi)=0\right\},
$$

hence $R$ can be naturally embedded into $\mathcal{M}(R)$ by the inclusion map $\iota: \xi \hookrightarrow M_{\xi}$. Since we already provided $\mathcal{M}(R)$ with the Gelfand topology, one may ask the following: "is $\iota(R)$ is dense in $\mathcal{M}(R)$ with respect to the Gelfand topology?" This is a famous question that is known as the corona problem, and we will say that the corona theorem holds for $R$ if $\iota(R)$ is dense in $\mathcal{M}(R)$. Otherwise $R$ is said to have corona $(=\mathcal{M}(R) \backslash \overline{\iota(R)})$. Note that the complex homomorphism $\varphi_{M_{\xi}}$ associated with $M_{\xi}$ is nothing but the point evaluation map $\lambda_{\xi}: f \mapsto f(\xi)$.

It is known that the corona theorem holds for $R$ if and only if the following function theoretical statement holds (see Chap. 4 of [9], Chap. VIII of [10, or Chap. 12 of [7]): for given $F_{1}, \ldots, F_{n} \in H^{\infty}(R)$ and $\delta \in(0,1)$ such that

$$
\delta \leq \max _{1 \leq j \leq n}\left|F_{j}(\zeta)\right| \leq 1 \quad \text { for all } \zeta \in R
$$

Received by the editors November 14, 2007.

2000 Mathematics Subject Classification. Primary 30H05, 30D55.

Key words and phrases. Corona problem, bounded analytic function.

This work was supported by the research fund of Hanyang University (HY-2007-000-0000-4844).

(C)2008 American Mathematical Society Reverts to public domain 28 years from publication 
there exist $G_{1}, \ldots, G_{n} \in H^{\infty}(R)$ that satisfy the equation

$$
F_{1} G_{1}+F_{2} G_{2}+\cdots+F_{n} G_{n}=1 .
$$

We refer to $F_{1}, \ldots, F_{n}$ as corona data of index $(n, \delta)$ and $G_{1}, \ldots, G_{n}$ as corona solutions associated with the given corona data. The constant

$$
C(n, \delta, R):=\sup \inf \max \left\{\left\|G_{1}\right\|_{\infty}, \ldots,\left\|G_{n}\right\|_{\infty}\right\}
$$

is called the "corona constant" of $R$, where the supremum is over all corona data satisfying (11) and the infimum is over all possible corona solutions associated with each set of corona data.

As usual, we interpret the infimum of an empty set as infinity; thus if a Riemann surface $R$ has corona, the corona constant $C(n, \delta, R)$ must be infinite for some index $(n, \delta)$. But what about the converse? If the corona theorem holds for $R$, is $C(n, \delta, R)$ finite for all indices $(n, \delta)$ ? The answer to this question is still unknown for general Riemann surfaces, but the answer is positive at least for finitely bordered Riemann surfaces, as the following theorem shows.

Theorem 1 (Hara and Nakai, [12]). For a given finitely bordered Riemann surface $R$, let $g(R)$ denote the genus of $R$ and $b(R)$ denote the number of boundary components of $R$. Then for each given index $(n, \delta)$ and numbers $g \in \mathbb{N} \cup\{0\}$ and $b \in \mathbb{N}$, we have

$$
\sup _{R \in \Re(g, b)} C(n, \delta, R)<\infty,
$$

where $\mathfrak{R}(g, b)$ is the collection of Riemann surfaces with $g(R)=g$ and $b(R)=b$.

The purpose of our paper is to give a short proof for this theorem. Note that Theorem 1 implies that one can find an upper bound of the corona constant of a finitely bordered Riemann surface that depends only on the index, the genus of $R$ and the number of boundary components of $R$. The case $g=0$ was proved by Gamelin in [8, and the case $g=0$ and $b=1$ is nothing but the famous Carleson's corona theorem for the unit disc [6].

There are various planar domains and Riemann surfaces for which the corona theorem holds ([6], 8], [11, 14, [17, 2], 4], 5], and more). On the other hand, a relatively small number of Riemann surfaces are known to have corona. The first such example was constructed by Cole (Chap. 4 of [9]), which was recently reconstructed in a simpler way in [16], and other Riemann surfaces that have corona can be found in 3 , and $[13$.

The corona problem for general planar domains is still open, and the answer is also unknown for a polydisc or a unit ball in $\mathbb{C}^{n}, n \geq 2$.

\section{Proof of Theorem 1}

Our proof is based on the following three theorems and the Carleson's corona theorem for the unit disc.

Theorem 2 (Mitsuru Nakai, [15]). Let $R$ and $R^{\prime}$ be Riemann surfaces and let $f: R^{\prime} \rightarrow R$ be an $m$-sheeted branched covering map for some $m<\infty$. Then the corona theorem holds for $R^{\prime}$ if and only if it holds for $R$.

In Theorem 2 Nakai considered only Riemann surfaces, that is, he considered only connected surfaces $R$ and $R^{\prime}$. However, one can check that the argument is 
still valid even when the surfaces are not connected; i.e., in Theorem 2 one can replace $R$ and $R^{\prime}$ by disjoint unions of Riemann surfaces.

Let $\mathbb{D}$ denote the unit disc. The next argument we need for the proof of Theorem 1 is the following.

Theorem 3 (Ahlfors, 1 1). Suppose $R$ is a finitely bordered Riemann surface with $g(R)=g$ and $b(R)=b$. Then there exists an $m$-sheeted branched covering map $f: R \rightarrow \mathbb{D}$, called the Ahlfors map, such that $b \leq m \leq 2 g+b$.

The last ingredient of our recipe is the following statement:

Theorem 4. Let $\left\{R_{j}\right\}$ be a sequence of Riemann surfaces. Then

$$
\sup _{j} C\left(n, \delta, R_{j}\right)<\infty
$$

for every index $(n, \delta)$ if and only if the corona theorem holds for $\bigsqcup_{j} R_{j}$, the disjoint union of $R_{j}$.

Proof. This theorem is essentially Lemma 3.1 of $[8$. In fact, in [8] the theorem was stated only for planar domains, but one can easily check that the proof is valid for our case.

Now we are ready to prove Theorem 1 .

Proof of Theorem 1. Suppose Theorem 1 is not true. Then there exist an index $\left(n_{0}, \delta_{0}\right)$ and a sequence of finitely bordered Riemann surfaces $\left\{R_{j}\right\}$ with $g\left(R_{j}\right)=g$ and $b\left(R_{j}\right)=b, j=1,2, \ldots$, such that

$$
C\left(n_{0}, \delta_{0}, R_{j}\right) \rightarrow \infty
$$

as $j \rightarrow \infty$. Furthermore, by Theorem 3 we can find $m_{j}$-sheeted branched covering maps $h_{j}: R_{j} \rightarrow D_{j}:=\{z:|z-3 j|<1\}$ with $b \leq m_{j} \leq 2 g+b$ for all $j$. However, by passing to a subsequence if necessary, we may assume that all the $m_{j}$ 's are the same, that is, there exists a constant $m$ such that $m=m_{j}$ for all $j$. Now let $D=\bigcup_{j} D_{j}$ and $\mathcal{R}=\bigsqcup_{j} R_{j}$.

Carleson's corona theorem for the unit disc [6] (Theorem 1 for the case $g=0$ and $b=1)$ implies that $\sup _{j} C\left(n, \delta, D_{j}\right)<\infty$ for any index $(n, \delta)$, thus the corona theorem for $D$ follows from Theorem 4 . Then by Nakai's theorem (Theorem 2), we see that the corona theorem also holds for $\mathcal{R}$, because the map $h: \mathcal{R} \rightarrow D$ defined by $\left.h\right|_{R_{j}}=h_{j}$ is an $m$-sheeted branched covering. According to Theorem 4 , however, $\mathcal{R}=\bigsqcup_{j} R_{j}$ must have corona, because $\sup _{j} C\left(n_{0}, \delta_{0}, R_{j}\right)=\infty$ by (2). This contradiction completes the proof of Theorem 1 .

We believe that what makes our proof significantly shorter than the proof of Hara and Nakai is the idea of applying Hara's theorem (Theorem 2) to disjoint sets, which is in fact due to Gamelin as Theorem 4 indicates. The rest of the proof is not very far from the original in the sense that both proofs use Ahlfors maps and Nakai's theorem [15] (Theorems 3 and 2 above).

\section{REFERENCES}

[1] Lars L. Ahlfors, Open Riemann surfaces and extremal problems on compact subregions, Comment. Math. Helv. 24 (1950), 100-134. MR0036318(12:90b)

[2] N. Alling, A proof of the corona conjecture for finite open Riemann surfaces, Bull. Amer. Math. Soc. 70 (1964), 110-112. MR0156967 (28:209) 
[3] D. E. Barrett and J. Diller, A new construction of Riemann surfaces with corona, J. Geom. Anal. 8 (1998), 341-347. MR1707732 (2000j:30076)

[4] M. Behrens, The corona conjecture for a class of infinitely connected domains, Bull. Amer. Math. Soc. 76 (1970), 387-391. MR0256166 (41:825)

[5] M. Behrens, The maximal ideal space of algebras of bounded analytic functions on infinitely connected domains, Trans. Amer. Math. Soc. 161 (1971), 359-379. MR0435420 (55:8380)

[6] L. Carleson, Interpolations by bounded analytic functions and the corona problem, Ann. of Math. (2) 76 (1962), 547-559. MR0141789 (25:5186)

[7] Peter L. Duren, Theory of $H^{p}$ spaces, Pure and Applied Mathematics 38, Academic Press, New York-London, 1970. MR0268655 (42:3552)

[8] T. W. Gamelin, Localization of the corona problem, Pacific J. Math. 34 (1970), 73-81. MR.0276742 (43:2482)

[9] T. W. Gamelin, Uniform algebras and Jensen measures, London Mathematical Society Lecture Note Series 32, Cambridge University Press, Cambridge-New York, 1978. MR521440 (81a:46058)

[10] J. B. Garnett, Bounded analytic functions, Pure and Applied Mathematics 96, Academic Press, Inc., New York-London, 1981. MR628971 (83g:30037)

[11] J. B. Garnett and P. W. Jones, The Corona theorem for Denjoy domains, Acta Math. 155 (1985), 27-40. MR793236 (87e:30044)

[12] Masaru Hara and Mitsuru Nakai, Corona theorem with bounds for finitely sheeted disks, Tohoku Math. J. (2) 37 (1985), no. 2, 225-240. MR788130 (86h:30079)

[13] M. Hayashi, Bounded analytic functions on Riemann surfaces, in: Aspects of complex analysis, differential geometry, mathematical physics and applications (St. Konstantin, 1998), World Sci. Publishing, River Edge, NJ, 1999, 45-59. MR.1731200 (2001i:30045)

[14] P. Jones and D. Marshall, Critical points of Green's functions, harmonic measure and the corona problem, Ark. Mat. 23 (1985), 281-314. MR827347 (87h:30101)

[15] Mitsuru Nakai, The corona problem on finitely sheeted covering surfaces, Nagoya Math. J. 92 (1983), 163-173. MR726148 (86b:30072)

[16] Byung-Geun Oh, An explicit example of Riemann surfaces with large bounds on the corona solutions, Pacific J. Math. 228 (2006), no. 2, 297-304. MR.2274522 (2007h:30053)

[17] E. L. Stout, Bounded holomorphic functions on finite Riemann surfaces, Trans. Amer. Math. Soc. 120 (1965), 255-285. MR0183882 (32:1358)

Department of Mathematics Education, Hanyang University, 17 Haengdang-dong, SeOngdong-Gu, Seoul 133-791, Korea

E-mail address: bgoh@hanyang.ac.kr 